\title{
THE PERSON-ORIENTED APPROACH IN THE FIELD OF EDUCATIONAL PSYCHOLOGY
}

\author{
Diana Raufelder, Danilo Jagenow, Frances Hoferichter \\ Free University of Berlin, Germany \\ E-mail: diana.raufelder@fu-berlin.de, danilo.jagenow@fu-berlin.de, \\ frances.hoferichter@fu-berlin.de
}

\author{
Kate Mills Drury \\ Concordia University, Montréal, Canada \\ E-mail: katemillsdrury@gmail.com
}

\begin{abstract}
Individual differences are a fundamental component of psychology, but these differences are often treated as "noise" or "errors" in variable-oriented statistical analyses. Currently, there is a small but emerging body of research using the person-oriented approach. In this paper a brief theoretical and methodological overview of the person-oriented approach is given. A person-oriented approach is often preferable where the main theoretical and analytical unit is a pattern of operating factors, rather than individual variables. In order to illustrate the relevance of this approach to research in educational psychology several representative statistical methods are outlined, two of which employ a person-oriented approach (latent class analysis/ latent profile analysis, configural frequency analysis/ prediction configural frequency analysis) and one that combines person and variable-oriented approaches. Examples of data analyses are used to demonstrate that variable and personoriented approaches provide the researcher with different information that can be complementary.
\end{abstract}

Key words: configural frequency analysis, educational psychology, individual differences, latent class analysis, person-oriented approach.

\section{Introduction}

Psychology as a discipline strives to balance idiographic information and nomothetic observation, however as we will see, one side of this dialectic is often emphasized at the expense of the other. During the last century the idiographic goal of describing the individual as a unique agent with a unique life history with properties setting one apart from other individuals, was widely substituted for the nomothetic perspective, which largely entails the search for laws that explain the generalities of objective phenomena. At least since the cognitive revolution and the emergence of the dimension-mathematics-experiment paradigm in the last century, research in psychology has been dominated by the variable-oriented approach, which focuses on measurement, quantification, and objective statistical methods. One reason for the pendulum swing was that the former idiographic perspective was fraught with critiques of subjectivity, weak data and measurement, and difficulties in theory testing (Bergman, \& Andersson, 2010). The paradigm shift brought with it vitalization and an explosion of knowledge within the field (Bergman \& Andersson, 2010) and has led to a broader acceptance of psychology as a serious discipline within the scientific community (Ittel \& Raufelder, 2008). However, the transformation of the field was not without its disadvantages: adopting a no- 
mothetic approach does not alter the fact that individual differences are a fundamental component of psychology, however, within the framework of variable-oriented statistical analyses these differences are often treated as "noise" or "errors" (Hampson \& Coleman, 1995). Consequently, inter-individual differences are often considered random and thus negligible (von Eye, Bogat, \& Rhodes, 2006) and intra-individual differences, based on a whole-system perspective (Bergman \& Andersson, 2010), in which the individual is seen as an organized whole (Bergman \& Magnusson, 1997), have been neglected to a surprising extent. Hampson and Coleman (1995) remind us that:

"One of the most important ways in which psychology differs from the natural sciences arises from the existence of individual differences. Two liters of hydrogen that are treated identically respond identically, but any two human beings, even identical twins, may respond quite differently to the same stimulus. This is because people differ from one another not only in appearance (that is, physically) but also in their behavior (that is, psychologically). Consequently, the study of individual differences, which encompasses personality, has been a significant part of psychology since ancient times" (p.X).

In 2004 Molenaar implored researchers to consider inter-individual differences and as such re-introduce the individual into psychological research. Today, there is a small but emerging body of research using the person-oriented approach in developmental psychology, signaling a pendulum swing back towards the idiographic perspective, in which the individual is regarded as a dynamic system of interwoven components (Bergman \& Andersson, 2010) and as an organized whole (Magnusson, 1990), functioning and developing as a totality (Bergman \& Magnusson, 1997) different from other individuals. This article is in response to Molenaar's (2004) appeal and gives a brief theoretical and methodological overview of the person-oriented approach and its implications for research in educational psychology.

\section{Individual Differences and the Person-Oriented Approach}

Individual differences are essential to psychology and especially to the field of educational psychology wherein the basic assumption that each individual learns in the same way under the same conditions can never be true. The earliest philosophers and humanists emphasized the need to identify student interests and adapt instruction to individual needs and differences, and the advantages of using self-comparisons rather than competitive social comparisons in evaluations of student's work and progress (Woolfolk, 2001). Crow and Crow (1973) remind us that: "educational psychology describes and explains the learning experiences of an individual from birth through old age" (p. 7). Furthermore, each person has an individual profile of characteristics, abilities and challenges that result from learning and their unique developmental history. These manifest as individual differences in intelligence, creativity, cognitive style, motivation, and the capacity to process information, communicate, and relate to others (Woolfolk, Winne, \& Perry, 2006). However, as a consequence of the dominance of variable-oriented statistical analyses, which assume equality between individuals, and a seeming reluctance to employ person-oriented methods (Rosato \& Bear, 2012), our knowledge about individual differences in educational psychology is limited.

One could argue that individual differences are considered in the discipline of differential psychology, which aims to identify the formal laws of variability (von Eye, 2010), but a basic assumption of research in differential psychology is that everybody can be assigned a location on the scales used for comparison (von Eye \& Spiel, 2010). In contrast, person-oriented research goes a step beyond this approach by acknowledging that particular concepts exist in or only apply to particular populations or even individuals. This basic tenet of the approach allows for the use of terms that are specific to populations, age groups, locations or historical times in the formulation of person-oriented theories. Furthermore, methodologically speaking, this tenet allows for the comparison of individuals based on the possibly changing structure of behavior domains, as well as based on the existence of behavioral domains (see von Eye \& Spiel, 2010), as opposed to comparing individuals solely on their location on particular scales. In other words, person-oriented research does not proceed from the assumption that the validity of concepts and variables is universal (see von Eye, 2009, 2010). Instead, one of the fundamental tenets underlying the person-oriented approach (Bergman \& Mag- 
nusson, 1997; Bergman, von Eye, \& Magnusson, 2006; von Eye \& Bergman, 2003) and idiographic psychology (Molenaar, 2004; Molenaar \& Campbell, 2009; von Eye, 2004) states that premature aggregation of data can result in conclusions that fail to do justice to the variability in populations (von Eye \& Spiel, 2010). Despite this principle, theoretical and methodological discussions within the person-oriented approach continue to proceed with the implicit assumption that the scales and measures used to describe individuals are universally valid (see von Eye \& Spiel, 2010). In order to better understand these persistent assumptions, the next paragraph gives a short overview of the constituent characteristics of the person-oriented approach.

\section{Characteristics of the Person-Oriented Approach}

Before describing the constituent characteristics of the person-oriented approach, it should be noted that over the years the term "person-oriented" (often used interchangeably with the terms "person-centered" and "pattern-oriented") has acquired many different meanings (Bergman \& Andersson, 2010). Additionally, some researchers do not distinguish between person-oriented theory and person-oriented methodology (Sterba \& Bauer, 2010) and label their approach as "person-oriented" or "person-centered" if some kind of pattern analysis has been made, even within a variable-oriented framework (Bergman \& Andersson, 2010). However, not every statistical analysis, which focuses the individual, is automatically person-oriented. Bergman and Andersson (2010) underline: "To a reasonable extent, the integrity of the system under study must also be retained" (p. 162). Due to the fact that to date there is no agreed upon single definition of a person-centered approach, this article summarizes the most common current perspectives on theory as well as methodology developed over the past thirty years and influenced heavily by the works of Bergman and Magnusson (Magnusson, 1988; Magnusson \& Törestad, 1993; Bergman \& Magnusson, 1997; Bergman, von Eye, \& Magnusson, 2006).

The original six tenets (theoretical elements) of the person-oriented approach (Bergman, 2001; Bergman \& Magnusson, 1997; von Eye \& Bergman, 2003) were adapted by Sterba and Bauer (2010) as the following person-oriented principles: (1) The individual-specificity principle holds that structure and dynamics of behavior are at least partly specific to the individual. (2) The complex-interactions principle implies the consideration of many factors and their interrelations to embrace the complexity of behavior. (3) The interindividual-differences/intraindividual-change principle assumes a lawfulness and structure to intraindividual constancy and change as well as inter-individual differences in constancy and change. (4) The pattern-summary principle follows the idea that processes develop in a lawful way and can be described as patterns of involved factors. (5) The holism-principle states that the meaning of the involved factors results from the interactions between these factors. (6) Finally, the pattern-parsimony principle asserts that the number of different patterns is infinite, but that some patterns occur more frequently than others. Von Eye (2010) postulates a reformulation of this last principle into expectancies, such as expected numbers of patterns should be specified, based on a number of arguments or model assumptions. The advantage of this approach is to test hypotheses concerning the observed frequencies of these events, which at the same time lightens the emphasis on predominantly descriptive statements (see Bergman, Magnusson, \& El-Khouri, 2003). In order to develop the environmental components of person-oriented research Bogat (2009) proposes two additional tenets: (a) "the structure and dynamics of individual behavior are, at least in part, specific to the environment in which the individual lives and work" as well as (b) "validity is specific to individuals and environments" (Bogat, 2009, p. 32).

The central question, which arises from these theoretical principles and approaches, is how to transform them into analytical methods. Sterba and Bauer (2010) discussed which of these personoriented principles could be tested via four types of latent variable analyses for longitudinal data in developmental psychology: (1) Less-restrictive variable-oriented methods (e.g., latent growth curve model), (2) classification methods (e.g., latent class growth analysis; latent Markov model), (3) hybrid classifications methods (growth mixture models), and (4) single-subject methods (e.g., dynamic factor analysis) (for details see Sterba \& Bauer, 2010). Despite this classification system, there remains a degree of arbitrariness in determining whether a given principle can be tested within each analytic approach (Mun, Bates, \& Vaschillo, 2010). Although Mun, Bates and Vaschillo assert 
that there is currently an effort to better match theoretical concepts with analytical tools (Mun, Bates, \& Vaschillo, 2010), there is still much disagreement about the appropriate use of person-oriented research methods.

The next section discusses three statistical analyses that are often used in person-oriented research, that adhere to the precept of reintroducing the individual into psychological research (Molenaar, 2004) and that are also helpful in answering research questions in educational psychology. The discussion aims to shed light on some of the difficulties inherent in integrating person oriented theory and methodology.

\section{Methods of Analysis of Person-Oriented Research}

In general, person-oriented methods enable the researcher to identify important intra-individual and inter-individual differences and thus model distinct configurations of heterogeneity within a given sample (Rosato \& Baer, 2012). That means that individuals will be studied on the basis of their patterns of individual characteristics specific to the research question. It should be noted that such patterns can occur at different levels (from the molecular to the global) and that a single study can only address a few patterns. Bergman and Magnusson (1997) address the role of variables within this pattern-orientation: "It is sometimes objected that even the person-oriented approach is variable oriented because, for instance, in many of its applications, variables are used to construct profiles of individuals' scores which are then used in the statistical analysis. However, variables included in such an analysis have no meaning in themselves. They are considered only as components of the pattern under analysis and interpreted in relation to all the other variables considered simultaneously; the relevant aspect is the profile of scores" (Bergman \& Magnusson, 1997, p. 293).

The basic goal in person-oriented research is to group individuals into categories, with each one containing individuals who are similar to each other and different from individuals in other categories (Muthén \& Muthén, 2000). Von Eye and Bogat (2006) defined three criteria for personoriented research: (1) a sample is analyzed under the assumption that it was drawn from more than one population, (2) attempts be made to establish external validity of subpopulations, and (3) groups be interpreted based on theory. These general criteria, as well as the methodological issues raised by Sterba and Bauer (2010), Molenaar (2010), and Mun, Bates and Vaschillo (2010) presuppose that the scales, instruments, and measures used to identify differences in individual profiles and patterns are equally meaningful in all subpopulations and for all individuals, although the personoriented approach is generally "open to the assumption that particular concepts exist in or apply to particular populations or even individuals only" (von Eye \& Spiel, 2010, p. 153). These seemingly ambiguous assumptions can be better illustrated through an example: the statement that car drivers often feel pressured by other car drivers only makes sense for car drivers, and not for non car drivers (latter assumption). In contrast, if researchers want to compare Canadian and German students on motivation, they must be sure that Canadian and German students understand motivation in the same way (presupposition of equal meaning). Von Eye explicates this presupposition in his concept of dimensional identity (for detail see von Eye, 2010) and its implicit condition of commensurability (for detail see von Eye \& Spiel, 2010).

One popular analysis, which is often used in person-oriented research and is becoming more and more common in educational psychology, is latent class analysis (LCA) or latent profile analysis (LPA). However, as we shall see, both follow the assumption that the scales, instruments, and measures used for building the classes/profiles are equally meaningful for all possible subpopulations and all individuals.

\section{Latent Class Analysis (LCA) \& Latent Profile Analysis (LPA)}

Latent class analysis (LCA) and latent profile analysis (LPA) (Lazarsfeld \& Henry, 1968) are non-parametric statistical techniques based on the assumption that patterns among a set of observed variables are explained by an unmeasured latent variable with discrete classes (Collins \& Lanza, 2010; Lazarsfeld \& Henry, 1968; McCutcheon, 1987). They are multivariate methods used to identify latent subpopulations of individuals based on multiple observed measures (Lubke \& Muthén, 2005). The 
two forms of analysis use maximum likelihood estimation for the analysis of different types of indicators, LCA uses binary and LPA uses continuous indicators, and assume that the association between items can be explained by the existence of several latent classes/profiles. LCA and LPA examine individuals as a whole based on their patterns of observed characteristics (Bergman \& Magnusson, 1997). Within one class, individuals are assumed to have identical patterns of solution probabilities. Participants can be assigned to a class for which his or her assignment probability is the highest. LCA and LPA are conceptually related to cluster analysis. The advantages of these approaches over cluster analysis are that they are model based and as such generate probabilities for group membership. In other words, these models can be tested and their goodness of fit can be analyzed. The following example illustrates the relevance of LCA to the field of educational psychology.

Using LCA in educational psychology research: Variable-oriented research in educational psychology typically produces results framed in the following way: for most adolescent students, positive social relationships in school are supportive of academic achievement and scholastic motivation (Wentzel et al. 2010; Wentzel, 1998). Indeed it is important to understand the experiences of most students, but what about the other students? Although much is known about the respective roles of teacher and peer support in motivational outcomes, much less is known about students with relatively constant levels of academic achievement and motivation independent of teachers and/or classmates. Therefore, using a person-oriented approach, we tested the following research question with LCA: Are social relationships important for scholastic motivation for all students, or are there different motivation types, including a type which does not need any or only limited social support from teachers and/or classmates in order to be motivated? A LCA using self-report data from 1088 $7^{\text {th }}$ and $8^{\text {th }}$ grade students identified four different motivation types (MTs): (1) teacher-dependent MT, (2) peer-dependent MT, (3) teacher- and peer-dependent MT, (4) teacher- and peer-independent MT. The results underscore inter-individual differences in the ways students rely on teachers and peers as sources of motivation. In contrast, many schools still expect students to learn and behave in uniform ways and students who do not fit this pattern are often viewed as maladjusted instead of, as the results of this person-oriented research have shown, having different motivational needs. The proposed typology can be used to improve students' learning by furthering understanding of and building on their individual motivational needs (for details see Raufelder, Jagenow, Drury, \& Hoferichter, 2013).

For longitudinal or developmental data in which hypotheses focus on heterogeneity in developmental trajectories and differential impact of covariates, growth mixture modeling (GMM) can be used, which combines the strengths of growth curve modeling (a variable-oriented method) with those of latent class analysis (a person-oriented method) (Muthén \& Muthén, 2000). Another common statistical analysis in person-oriented research, configural frequency analysis (CFA), is often used for longitudinal analyses and is the subject of the next section.

\section{Configural Frequency Analysis (CFA) and Prediction Configural Frequency Analysis (P-CFA)}

One of the principle goals of person-oriented research is to make statements about individuals or homogenous groups of individuals (so-called cells) (von Eye, 1990, 2010). This goal can be achieved through CFA (Lienert \& Krauth, 1975; von Eye, 2002; von Eye \& Gutiérrez Peña, 2004). While methods such as log-linear modeling or logistic regression center around variables, CFA allows hypotheses about individual cells or groups of cells of cross-classifications (von Eye, 2010) to be tested. In general, configural frequency analysis is a multivariate statistical method that identifies individuals (configurations) that are unique in a statistically significant way. CFA aims to detect patterns in data that occur significantly more (such patterns are called type) or significantly less often (such patterns are called antitypes) than expected by chance. Whereas types are interpreted as concepts that are constituted by a pattern of variable values, antitypes are interpreted as patterns of variables that do not, in general, occur together (Lienert, 1969). The results of CFA are lists of type- or antitype-constituting configurations, which reflect local relationships in the data (local relationships refers to patterns of variable categories, but not necessarily to entire variables with all their categories) (von Eye \& Mun, 2012; Havránek \& Lienert, 1984). Whereas in CFA models are constructed with variables of the same status, Prediction CFA (P-CFA) distinguishes between 
predictor and criterion variables (von Eye \& Rovine, 1994): “Types and antitypes are identified as criterion attribute patterns that, given a certain predictor pattern, occur more (or less) often than estimated by some chance model" (von Eye \& Rovine, 1994, p. 2).

There are two underlying main approaches in CFA: (1) Identifying relationships among variables, which include coefficients of association that can be interpreted in a manner analogous to correlations and (2) identifying patterns of characteristics, which focus on groups of subjects. These groups contain subjects who differ from all other subjects in that they display a unique pattern of characteristics (configuration). In contrast to cluster analysis, CFA does not lead to solely descriptive statements; it goes a step beyond in providing descriptive (labels for a configuration) and inferential results (probability of the frequency of a given configuration relative to some expected frequency). Like LCA or LPA, CFA allows for the creation of groups that differ in size, which is essential from the perspective of person-oriented research (von Eye, Bogat, \& Rhodes, 2006). The following paragraphs underline the use of CFA/P-CFA for longitudinal data. In general, longitudinal CFA/P-CFA focuses on the characteristics of transitions. The following example illustrates the suitability of longitudinal P-CFA for research in the field of educational psychology.

Using CFA in educational psychology research: The work of Stemmler and Lösel (2012) aims to identify approaches for preventing externalizing behaviors (such as aggression, delinquency, hyperactivity etc.) in educational settings. Therefore, understanding the persistence and aggravation of externalizing problems over time is essential to their research. Based on previous person-oriented analyses (Stemmler et al., 2005, 2008; Stemmler \& Lösel, 2010), in which two types (Type1: 'externalizing only', Type 2:'externalizing plus internalizing') of externalizing problems in boys were identified, the researchers investigated whether the groups remained stable over time and whether the two types of antisociality were related to offending in adolescence by using prediction-configural frequency analysis (P-CFA). The sample consisted of 295 boys from the Erlangen-Nuremberg Development and Prevention Study (Lösel, Stemmler, Jarusch, \& Beelmann, 2009). Social behavior was rated by mothers, kindergarten educators, and schoolteachers; offending was self-reported by the adolescents. The time lag between the first and last data assessment was more than eight years. In the P-CFA the 'externalizing only' pattern was replicated and suggested high stability over time. Moreover, this pattern was clearly related to self-reported delinquent behavior. In addition, the results of the P-CFA showed a three-way interaction between externalizing and internalizing variables, which could not have been found using a variable-oriented approach (Stemmler et al., 2005). Interestingly, the 'externalizing plus internalizing' pattern did not appear as a type, in contrast to Stemmler and Lösel's previous studies (for more details see Stemmler \& Lösel, 2012).

The growing knowledge of different developmental subtypes of problem behavior underlines once more the need for person-oriented research in educational psychology, which could lead to the development of individualized prevention and intervention initiatives. Alongside the abovementioned 'pure' person-oriented methods of analysis, we now find a trend towards integrating person-oriented and variable-oriented analyses in order to close the gap between the person- and the variable-oriented approaches. This integration is the next topic of discussion.

\section{Integrating Person-Oriented and Variable-Oriented Analyses}

By integrating person-oriented and variable-oriented analyses researchers attempt to minimize the weaknesses of each approach and maximize their strengths by combining disparate but complementary assumptions (variable-oriented vs. person-oriented statements). In such cases Bergman (1998) proposes the following sequence of analyses (see also von Eye, 2010): (1) Identifying operating factors by using exploratory, variable-oriented analyses (Feyerabend, 1975; von Eye \& Bogat, 2006); (2) Identifying possibly existing subpopulations by using exploratory, person-oriented analyses (von Eye \& Bogat, 2006); (3) Testing theoretical assumptions by using confirmatory person-oriented analyses of data from independent samples and; (4) Linking theories and results from the different research strategies by using variable-oriented analyses (Feyerabend, 1975; Molenaar \& Campbell, 2009). The advantage of these combined analyses is self-evident: While the person-oriented approach is useful in its ability to describe different experiences for 
different profiles of youth, it does not reveal associations between variables that are common to all youth. Therefore, by combining the methods researchers gain information about profiles of distinct groups as well as generalities across entire samples.

Using a combination of the variable-oriented and person-oriented approach in educational psychology research: Davidson, Gest and Welsh (2010) conducted a longitudinal study with 383 youth to examine relatedness with teachers and peers during early adolescence. In detail, they (a) identified patterns of early transition relatedness in the fall of the first year of middle school (6th grade); (b) examined how pre-transition behavior (fall of 5th grade) was associated with early transition relatedness; and (c) integrated variable- oriented and person-oriented approaches to study how indicators and patterns of early transition relatedness were associated with school adjustment (i.e., academic skills, academic self-concept, school bonding, loneliness, and selfworth), concurrently and one and a half year later (spring of 7th grade). Using LPA, three patterns of early transition teacher-peer relatedness were identified: "Profile $1(n=168)$ was characterized by low teacher-student closeness, low peer social preference, and low perceived peer competence (Low Relatedness). Profile 2 ( $n=93$ ) was characterized by average teacher-student closeness and high peer social preference and perceived peer competence (peer-oriented). Profile $3(n=122)$ was characterized by high teacher-student closeness, high peer social preference, and high perceived peer competence" (Davidson, Gest, \& welsh, 2010, p. 495). Following these analyses two more methodological steps were taken: (1) multinominal logistic regression analysis was used to assess prediction of membership in one of the three early transition relatedness profiles based on adolescents' pre-transition behavior in fall of $5^{\text {th }}$ grade and (2) a series of hierarchical linear regression models were conducted to examine the unique and additive contribution of (a) the continuous indicators of early transition relatedness with teachers and peers and (b) the early transition relatedness profiles on youths' adjustment in the fall of 6th grade and the spring of 7 th grade (for details see Davidson, Gest, \& Welsh, 2010). Findings indicated that behavioral characteristics in elementary school might contribute to early transition patterns of relatedness with teachers and peers in middle school. Furthermore, findings showed that a pattern of poor relationships with primary social partners in the school context indicated youth at risk for maladjustment.

In line with the other above-mentioned examples of person-oriented research, the use of LPA allowed for the identification of unique patterns of teacher-peer relatedness. Additionally, the combination of variable-oriented with person-oriented methods enabled the researchers to also identify patterns of association with earlier behavioral characteristics and concurrent adjustment above and beyond the independent indicators of relatedness. These results underline how experiences with teachers and peers are meaningful in different ways for different youth and we believe that this rich and nuanced information better serves teachers and educators dedicated to ameliorating educational experiences for adolescents.

There are other methods of analysis, which can be used in person-oriented research; some of them have yet to find a concrete application such as comparative methods (Caramani, 2009) or symbolic data analysis (Billard \& Diday, 2006) but others have been used extensively in the field of developmental psychology such as latent growth curve model (LGM), latent class growth analysis (LCGA), and latent Markov model (e.g., Sterba \& Bauer, 2010; Bergman \& Magnusson, 1997). An interesting area for future discussion would be the degree to which multilevel analyses in educational psychology can be understood as person-oriented analyses within variable-oriented research, seeing as school level, class level and individual student level analyses can be conducted simultaneously.

In sum, as the three outlined analysis techniques have shown, person-oriented research is essential to the field of educational psychology. The fact that individuals differ in abilities, capacities and personality characteristics as well as in their personal development necessitates the adoption of individual-centered perspectives in educational settings. It seems as though Crow and Crow's assertion from 1973 is still germane to current education policy: "Since we supposedly are teaching individuals, not groups of individuals, it is the function of the school within its budgetary personnel and curricular limitations to provide adequate schooling for every learner no matter how much he differs from every other learner" (p. 215). 


\section{Conclusions}

Inter-individual and intra-individual differences are at the heart of educational psychology, which as a discipline concerns itself primarily with individual learning processes. Nevertheless, research in educational psychology (as in psychology general) has been dominated by variable-oriented research for decades. Results of variable-oriented research often provide information about students, children and adolescents on average, which does not allow for the implementation of learning support on an individual level. In contrast, the person-oriented approach, which has been developed in the field of developmental psychology, explicitly addresses inter-individual as well as intra-individual differences. By outlining the theoretical and methodological characteristics of the person-oriented approach, the problems associated with matching the theoretical tenets of the approach with appropriate methods of analysis, as well as the implications of the approach for educational psychology, this paper highlights the urgent need to integrate person-oriented theory and methodology into educational psychology research.

\section{Acknowledgements}

This article was supported by a grant from the Volkswagen Foundation.

\section{References}

Bergman, L. R. (1998). A pattern-oriented approach to studying individual development: Snapshots and processes. In R.B. Cairns, L.R. Bergman, \& J. Kagan (Eds.), Methods and models for studying the individual (pp. 83-122). Thousand Oaks, CA: Sage.

Bergman, L. R. (2001). A person approach in research on adolescence: some methodological challenges. Journal of Adolescence Research, 16, 28-53.

Bergman, L. R., \& Andersson, H. (2010). The person and the variable in developmental psychology. Journal of Psychology, 218, 155-165.

Bergman, L. R., \& Magnusson, D. (1997). A person-oriented approach in research on developmental psychopathology. Development and Psychopathology, 9, 291-319.

Bergman, L. R., Magnusson, D., \& El-Khouri, B. M. (2003). Studying individual development in an interindividual context. Mahwah, NJ: Erlbaum.

Bergman, L. R., von Eye, A., \& Magnusson, D. (2006). Person-oriented research strategies in developmental psychopathology. In D. Cicchetti \& D. J. Cohen (Eds.), Developmental psychopathology (pp. 850-888) (2nd ed.). London, UK: Wiley.

Bogat, G. A. (2009). Is it necessary to discuss person-oriented research in community psychology? American Journal of Community Psychology, 43, 22-34.

Collins, L. K., \& Lanza, S. T. (2010). Latent class and latent transitional analysis: with applications in the social, behavioral, and health sciences. Hoboken, NJ: Wiley \& Sons.

Crow, L. D., \& Crow, A. (1973). Educational Psychology. New York, NY: Eurasia.

Davidson, A. J., Gest, S. D., \& Welsh, J. A. (2010). Relatedness with teachers and peers during early adolescence: An integrated variable-oriented and person-oriented approach. Journal of School Psychology, 48, 483-510.

Feyerabend, P. (1975). Against method. London, UK: Wiley.

Hampson, S. E., \& Colman, A. M. (Eds.) (1995). Individual differences and personality. London, GB: Longman.

Havránek, T., \& Lienert, G. A. (1984). Local and regional versus global contingency testing. Biometrical Journal, 26, 483 - 494.

Ittel, A. \& Raufelder, D. (2008). Lehrer und Schüler als Bildungspartner. Ansätze zwischen Tradition und Moderne. Göttingen: Vandenhoeck \& Ruprecht.

Lazarsfeld, P. F., \& Henry, N. W. (1968). Latente Structure Analysis. Boston: Houghton Mifflin.

Lienert, G.A. (1969). Die Konfigurationsfrequenzanalyse als Klassifikationsmethode in der klinischen Psy- 
chologie [Configural frequency analysis as a classification method in clinical psychology]. In Irle, M. (Ed.), Bericht über den 26. Kongress der Deutschen Gesellschaft für Psychologie in Tübingen 1968 (p. 244-253). Göttingen: Hogrefe.

Lienert, G. A., \& Krauth, J. (1975). Configural frequency analysis as statistical tool for defining types. Educational and Psychological Measurement, 35, 231-238.

Lösel, F., Stemmler, M., Jaursch, S., \& Beelmann, A. (2009). Universal prevention of anti social development: Short- and long-term effects of a child and parent-oriented program. Monatsschrift für Kriminologie und Strafrechtsreform, 92, 289-308.

Lubke, G. H., \& Muthén, B. (2005). Investigating Population Heterogeneity With Factor Mixture Models. Psychological Methods, 10, 21-39.

Magnusson, D. (1988). Individual development from an interactional perspective: A longitudinal study. Hillsdale, NJ: Erlbaum.

Magnusson, D. (1990). Personality development from an interactional perspective. In L. Pervin (Ed.), Handbook of personality: Theory and research (pp. 193-222). New York, NY: Guilford.

Magnusson, D., \& Törestad, B. (1993). A holistic view of personality: A model revisited. Annual Review of Psychology, 44, 427-452.

McCutcheon, A. L. (1987). Latent class analysis. New York, NY: Houghton.

Molenaar, P. C. M. (2004). A manifesto on Psychology as idiographic science: Bringing the person back into scientific psychology - This time forever. Measurement: Interdisciplinary Research and Perspectives, 2, 201-218.

Molenaar, P. C. M., \& Campbell, C. G. (2009). The new person-specific paradigm in psychology. Current Directions in Psychological Science, 18, 112-117.

Mun, E. Y., Bates, M. E., \& Vaschillo, E. (2010). Closing the gap between person-oriented theory and methods. Development and Psychopathology, 22, 261-271.

Muthén, B. O., \& Muthén, L. K. (2000). Integrating Person-Centered and Variable-Centered Analyses: Growth Mixture Modeling With Latent Trajectory Classes. Alcoholism: Clinical and Experimental Research, 24, 882-891.

Muthén, L. K., \& Muthén, B. O. (2010). Mplus User's Guide. Sixth Edition. Muthén \& Muthén, Los Angeles, CA.

Raufelder, D., Jagenow, D., Drury, K. \& Hoferichter, F. (2013). Social Relationships and Motivation in Secondary School: 4 different motivation types. Learning and Individual Differences, 22, (in press) doi:10.1016/j.lindif.2012.12.002

Rosato, N. S. \& Baer, J. C. (2012). Latent class analysis: a method for capturing heterogeneity. Social Work Research, 36, 61-69. doi: 10.1093/swr/svs006

Sterba, S. K., \& Bauer, D. J. (2010). Matching method with theory in person-oriented developmental psychopathology research. Development and Psychopathology, 22, 239-254.

Stemmler, M., Lösel, F., Beelmann, A., Jaursch, S., \& Zenkert, B. (2005). Child problem behavior in kindergarten and in primary school: A comparison between prediction con- figural frequency analysis and multiple regression. Psychology Science, 47, 467-478.

Stemmler, M. Lösel, F., Beelmann, A., \& Jaursch, S. (2008). A configural perspective on the stability of externalizing problem behavior in children: Results from the Erlangen- Nuremberg Development and Prevention Study. In M. Stemmler, E. Lautsch \& D. Mar- tinke (Eds.), Configural Frequency Analysis (CFA) and other non-parametrical statistical methods: Gustav A. Lienert Memorial issue (pp. 70-83). Lengerich: Pabst Science Publishers.

Stemmler, M., \& Lösel, F. (2010). Different patterns of boys' externalizing behavior and their relation to risk factors: A longitudinal study of preschool children. Bulletin de la Société des Sciences Médicales Luxembourg, 10, 53-62.

Stemmler, M., \& Lösel, F. (2012). The stability of externalizing behavior in boys from preschool age to adolescence: A person-oriented analysis. Psychological Test and Assessment Modeling, 54, 195-207.

von Eye, A., \& Rovine, M. J. (1994). Non-standard log-linear models for orthogonal prediction Configural Frequency Analysis. Biometrical Journal, 36, 177-184.

von Eye, A., \& Bergman, L. R. (2003). Research strategies in developmental psychopathology: Dimensional 
identity and the person-oriented approach. Development and Psychopathology, 15, 553-580.

von Eye, A., \& Gutiérrez Peña, E. (2004). Configural frequency analysis - the search for extreme cells. Journal of Applied Statistics, 31, 981-997.

von Eye, A., \& Bogat, G. A. (2006). Person orientation - concepts, results and development. Merrill Palmer Quarterly, 52, 390-420.

von Eye, A., Bogat, A., Rhodes, J. E. (2006). Variable-oriented and person-oriented perspective of analysis: the example of alcohol consumption in adolescence. Journal of Adolescence, 29, 981-1004. doi: 10.1016/j. adolescence.2006.06.007

von Eye, A. \& Spiel, C. (2010). Conducting person-oriented research. Zeitschrift für Psychologie/ Journal of Psychology, 218, 151-154. Doi: 10.1027/00443409/a000024

Von Eye, A., \& Mun, E. Y. (2012). Interindividual differences in intraidividual change in categorical variables. Psychological Test and Assessment Modeling, 54, 151-167.

von Eye, A. (2002). Configural frequency analysis - methods, models, and applications. Mahwah, NJ: Erlbaum.

von Eye, A. (2004). The treasures of Pandora's box. Measurement: Interdisciplinary Research and Perspectives, 2, 244-247.

von Eye, A. (2009). Universals and individuals - Is this the end of the discussion? Measurement: Interdisciplinary Research \& Perspective, 7, 3-7.

von Eye, A. (2010). Developing the person-oriented approach - Theory and methods of analysis. Development and Psychopathology, 22, 277-285.

Wentzel, K. R. (1998). Social relationships and motivation in middle school: the role of parents, teachers and peers. Journal of Educational Psychology, 90, 202-209.

Wentzel, K. R., Battle, A., Russell, S. L., \& Looney, L. B. (2010). Social supports from teachers and peers as predictors of academic and social motivation. Contemporary Educational Psychology, 35, 193-202.

Woolfolk, A. (2001). Educational Psychology, 8th edition. Boston, MA: Allyn and Bacon.

Woolfolk, A., Winne, P. H. \& Perry, N. E. (2006). Educational Psychology (3rd Canadian ed.). Toronto, Canada: Pearson.

Advised by Renata Bilbokaite,, University of Siauliai, Lithuania

Received: December 16, 2012

Accepted: March 08, 2013

\begin{tabular}{cl}
\hline Diana Raufelder & $\begin{array}{l}\text { Dr. phil., Assistant Professor, Free University Berlin, Habelschwerdter Allee 45, } 14195 \\
\text { Berlin, Germany. } \\
\text { E-mail: diana.raufelder@fu-berlin.de } \\
\text { Website: http://www.self-project.de }\end{array}$ \\
\hline \multirow{2}{*}{ Danilo Jagenow } & Dipl. Psych., Doctoral Candidate, Free University Berlin, Habelschwerdter Allee 45, 14195 \\
& Berlin. \\
& E-mail: danilo.jagenow@fu-berlin.de \\
& Website: http://www.self-project.de \\
\hline \multirow{2}{*}{ Frances Hoferichter } & M.A., Doctoral Candidate, Free University Berlin, Habelschwerdter Allee 45, 14195 Berlin, \\
& Germany. \\
& E-mail: frances.hoferichter@fu-berlin.de \\
& Website: http://www.self-project.de \\
\hline \multirow{2}{*}{ Kate Drury } & M.A., Doctoral Candidate, Concordia University Montréal, 7141 Sherbrooke Street West, \\
& H4B 1R6 Montréal, Québec, Canada. \\
& E-mail: katemillsdrury@gmail.com \\
Website: http://crdh.concordia.ca/bukowskilab/Bukowski_Lab_Site/Kate-Mills_Drury.html
\end{tabular}

